IPM/P-2006/013

TIFR/TH/06-06

\title{
Partition function of non-supersymmetric black holes in the supergravity limit
}

\author{
Shahrokh Parvizi ${ }^{a 1}$ and Alireza Tavanfar ${ }^{a, b} 2$ \\ ${ }^{a}$ Institute for Studies in Theoretical Physics and Mathematics (IPM) \\ P.O. Box 19395-5531, Tehran, Iran \\ ${ }^{b}$ Tata Institute of Fundamental Research \\ Homi Bhabha Road, Mumbai, 400 005, INDIA
}

\begin{abstract}
In this note, we propose the free energy of general non-supersymmetric black hole attractors arising in type IIA(B) superstrings on 3-fold Calabi-Yau, in the supergravity limit. This, by definition, differs from its counterpart BPS free energy by a factor of 4 . Correspondingly, a mixed ensemble for these black holes is proposed.
\end{abstract}

\footnotetext{
${ }^{1}$ parvizi@theory.ipm.ac.ir

${ }^{2}$ art@ipm.ir
} 


\section{Introduction}

Non-supersymmetric black holes are interesting phenomenologically but hard to handle theoretically. Recently due to the discovery of non-supersymmetric attractors $[1-4]^{3}$, new perspectives welcome exploring such black holes. These attractors can be black hole solutions to either a non-supersymmetric theory of gravity coupled to the gauge fields and moduli fields $[2,7]$, or non-BPS solutions to a supersymmetric theory $[3,8]$. In this note we are interested to some examples of the second class. More precisely, following the studies of $[3,8]$, we consider non-supersymmetric black hole attractors which arise in $C Y_{3}$ compactifications of type IIA(B) superstrings, where the effective theory is $\mathcal{N}=2$ supersymmetric and enjoys the well-developed structure of symplectic geometry, in the large volume Calabi-Yau and large charge limit, where the prepotential is approximated by its zero-genus term,

$$
F(X)=D_{i j k} \frac{X^{i} X^{j} X^{k}}{X^{0}} \quad ; \quad i, j, k=1 . . n
$$

To be an attractor, a non-supersymmetric black hole in this theory should be extremal, [2], and should not carry the D6-brane charge, [3]. This note concerns attractors and so these two requirements are assumed to be the case from now on. In $[3,8]$ the corresponding attractor solution are found. Then, one question which is natural to be addressed is evaluating the partition function of these black holes. This is the subject of this note. Given the black hole charge-multiplet $\left(p^{i}, q_{i}, q_{0}\right)$, we shall identify the mixed partition function of such non-susy black hole as,

$$
Z_{n s . B H} \equiv \sum_{M} \mathrm{~d}[\mathrm{D}(\mathrm{p}) \mathrm{M}] \mathrm{e}^{-\mathrm{M} \Im\left[\mathrm{CX}^{0}\right]}=\mathrm{e}^{\frac{\pi}{2} \frac{\mathrm{D}(\mathrm{p})}{\Im\left[\mathrm{CX} \mathrm{X}^{0}\right]}}
$$

with

$$
M=-2 \pi \hat{q}_{0}
$$

$\hat{q}_{0} \equiv q_{0}+\frac{1}{12} D^{i j}(p) q_{i} q_{j} ; D^{i j}(p) D_{j k}(p)=\delta_{k}^{i} ; D_{i j}(p) \equiv D_{i j k} p^{k} ; D(p) \equiv D_{i j k} p^{i} p^{j} p^{k}$

and $\mathrm{d}[\mathrm{D}(\mathrm{p}) \mathrm{M}]=\mathrm{d}(\mathrm{p}, \mathrm{q})$ denoting the degeneracy of black hole states given the charges. The way we find this result is similar to the analysis of [9], in essence. We first rewrite the general attractor equations of [8] in a slightly different form, which is more similar to the language of [9], as follows,

$$
\begin{aligned}
p^{I} & =\Re\left[C X^{I}-G^{j \bar{l}} \bar{\nabla}_{\bar{l}} \bar{C} \nabla_{j} X^{I}\right] \\
q_{I} & =\Re\left[C F_{I}-G^{j \bar{l}} \bar{\nabla}_{\bar{l}} \bar{C} \nabla_{j} F_{I}\right]
\end{aligned}
$$

\footnotetext{
${ }^{3}$ for earlier works see $[5,6]$.
} 


$$
2 C \partial_{i} \bar{C}-\mathbf{i} C_{i j k} G^{j \bar{n}} G^{k \bar{l}} \bar{\partial}_{\bar{n}} C \bar{\partial}_{\bar{l}} C=0
$$

At the next step we reformulate the original OSV ensemble, which is for BPS black holes, in a reduced but equivalent form. Then, motivated by this reformulation, we show that for the non-supersymmetric solution of (44,6), the black hole entropy is related via a Legendre transformation to the free energy,

$$
\mathcal{F}_{n s . B H}=\frac{\pi}{2} \frac{D(p)}{\Im\left[C X^{0}\right]}
$$

The Legendre transformation reds as,

$$
S_{n s . B H}(p, q)=\mathcal{F}_{n s . B H}-\Im\left(C X^{0}\right) \frac{\partial \mathcal{F}_{n s . B H}}{\partial \Im\left(C X^{0}\right)}
$$

with,

$$
\hat{q}_{0}=\frac{1}{2 \pi} \frac{\partial \mathcal{F}_{n s . B H}}{\partial \Im\left(C X^{0}\right)}
$$

The free energy (7) then supports for the mixed partition function given by (2). Here there are two points which we would like to highlight:

1. In the technical sense, the sum in (2) should be interpreted carefully. The problem is that $\mathrm{d}(\mathrm{p}, \mathrm{q})$ as derived by the inverse Laplace transformation corresponding to (2), does not necessarily respect the expected symplectic symmetry. The same problem is the case for the mixed OSV ensemble for which there are two ways to restore this symmetry. One way is incorporating the sum with an appropriate measure, for example [10-13] suggest such measures for the case of OSV ensemble. An equivalent way is based on the approach of [14]. One uses the fact that the Legendre transformation which bridges between the free energy (7) and the black hole entropy is preserved up to a rescaling of the 'potential' $\Im\left(C X^{0}\right)$. Then applying the symplectic symmetry as a constraint on $\mathrm{d}(\mathrm{p}, \mathrm{q})$, a proper charge-dependent rescaling of $\Im\left(C X^{0}\right)$ can be chosen to redefine the ensemble.

2. Unlike the main stream of [9], we do not connect (2) with the partition function of topological strings. This is because such a connection faces some serious conceptual difficulties ${ }^{4}$.

The outline of this work is as follows. In section 2 , we review the derivation of the general attractors, rewrite them in the form of (4,6) and recover the results of $[3,8]$. In Section 3, through 3 subsections, we first give a brief recap of the OSV proposal, then introduce an equivalent reduced form of the OSV ensemble and finally find the partition function of the non-supersymmetric attractors in the supergravity limit. We end this note with a short list of some open problems.

\footnotetext{
${ }^{4}$ We are thankful to Cumrun Vafa for discussion in this regard.
} 


\section{Attractor equations}

It is easier to tell the story in the IIB language. Mirror symmetry then takes us to the IIA dual. Let us begin with an arbitrary 3-cycle $\Gamma$ living in the 3-fold CalabiYau $X$ on which one compactifies the theory. In a symplectic basis $\left(A^{I}, B_{I}\right)$, with $I$ running from 0 to $n+1$, the unique decomposition of $\Gamma$ reads as,

$$
\Gamma=q_{I} A^{I}-p^{I} B_{I}
$$

The Poincare dual of this cycle is a 3 -form $G_{3}$, called the 3 -form flux, which in the dual basis $\left(\alpha_{I}, \beta^{I}\right)$ takes the form,

$$
G_{3}=p^{I} \alpha_{I}-q_{I} \beta^{I}
$$

In this flux background the GVW superpotential [15] is defined as,

$$
W \equiv \int G_{3} \wedge \Omega
$$

with $\Omega$ being the holomorphic 3-form of $X$. In terms of the symplectic period variables,

$$
X^{I} \equiv \int_{A^{I}} \Omega \quad ; \quad F_{I} \equiv \int_{B^{I}} \Omega=\partial_{I} F(X)
$$

the superpotential reads as,

$$
W=q_{I} X^{I}-p^{I} F_{I}
$$

Let further parametrize the complex structure moduli of $X$ by $t^{i} \equiv \frac{X^{i}}{X^{0}}, i=0 \ldots n$, and $\nabla_{i}$ stand for (the components of) the Kähler covariant derivative,

$$
\nabla_{i} X^{J} \equiv \partial_{i} X^{J}+\left(\partial_{i} K\right) X^{J} \quad ; \quad \bar{\nabla}_{\bar{i}} X^{J} \equiv 0
$$

corresponding to the Kähler potential,

$$
K=-\ln \left[\mathbf{i}\left(\overline{\mathrm{X}}^{\mathrm{J}} \mathrm{F}_{\mathrm{J}}-\mathrm{X}^{\mathrm{J}} \overline{\mathrm{F}}_{\mathrm{J}}\right)\right] .
$$

As explored in [16-19] for BPS cases, and further in [2-6,8] for non-BPS cases, the extremal black hole solution enjoys the attractor mechanism, according to which the moduli fields with free asymptotic values at infinity take some fixed values on the horizon, as determined by the black hole charges. More precisely, these attractor values are uniquely determined by minimizing an effective potential. In a theory with $\mathcal{N}=2$ supersmmetry, this effective potential is given in terms of the GVW superpotential as,

$$
V_{e f f} \equiv \mathrm{e}^{\mathrm{K}}\left(|\mathrm{W}|^{2}+\left|\nabla_{\mathrm{i}} \mathrm{W}\right|^{2}\right)
$$

The black hole is BPS if and only if,

$$
\nabla_{i} W=0
$$


which is the trivial (minimum) solution of

$$
\nabla_{i} V_{\text {eff }}=\partial_{i} V_{\text {eff }}=0
$$

Non-supersymmetric black holes correspond to non-trivial (minimum) solutions of (14). Recently $[3,8]$ solved these equations in the supergravity limit which corresponds to (11). They showed that for (11), equations (14) admit either a BPS solution if $\hat{q}_{0} D(p) \geq 0$ or a non-supersymmetric solution if $\hat{q}_{0} D(p) \leq 0$, with the convention (3). To get this result, [3] simply plugs (10) back into (14). [8] however develops a different approach which we report here briefly. Given $\Omega$, the Hodge-decomposition,

$$
H^{3}(X)=H^{3,0} \oplus H^{2,1} \oplus H^{1,2} \oplus H^{0,3}
$$

admits the basis,

$$
\Omega \oplus \nabla_{i} \Omega \oplus \bar{\nabla}_{\bar{i}} \bar{\Omega} \oplus \bar{\Omega} .
$$

In this basis, a real 3 -form like $G_{3}$ has the general expansion,

$$
G_{3}=\mathbf{i}\left[\bar{x} \bar{\Omega}+\bar{x}^{\bar{j}} \bar{\nabla}_{\bar{j}} \bar{\Omega}-\text { c.c. }\right] .
$$

Now taking $\int \Omega \wedge$ of both sides of (15) and using the identity,

$$
\mathbf{i} \int \Omega \wedge \bar{\Omega}=\mathrm{e}^{-\mathrm{K}}
$$

one gets $\bar{x}=-Z$, with $Z \equiv \mathrm{e}^{\mathrm{K} / 2} \mathrm{~W}$ defined as the (covariantly holomorphic) central charge. Similarly applying $\int \nabla_{l} \Omega \wedge$ to both sides of (15) and using,

$$
\text { i } \int \bar{\nabla}_{\bar{j}} \bar{\Omega} \wedge \nabla_{l} \Omega=\mathrm{e}^{-\mathrm{K}} \mathrm{G}_{\overline{\mathrm{j}}}
$$

one gets $\bar{x}^{\bar{j}}=G^{l \bar{j}} \nabla_{l} Z$. Thus the general expansion of the 3-form flux reads as,

$$
G_{3}=2 \mathrm{e}^{\mathrm{K}} \Im\left[\mathrm{W} \bar{\Omega}-\mathrm{G}^{\mathrm{l} \overline{\mathrm{j}}} \nabla_{\mathrm{l}} \mathrm{W} \bar{\nabla}_{\overline{\mathrm{j}}} \bar{\Omega}\right] .
$$

Subsequently integrating both sides of 16 over the cycles $\left(A^{I}, B_{I}\right)$ yields,

$$
p^{I}=2 \mathrm{e}^{\mathrm{K}} \Im\left[\mathrm{W} \overline{\mathrm{X}}^{\mathrm{I}}-\mathrm{G}^{\mathrm{lj}} \nabla_{\mathrm{l}} \mathrm{W} \bar{\nabla}_{\overline{\mathrm{j}}} \overline{\mathrm{X}}^{\mathrm{I}}\right]
$$

and

$$
q_{I}=2 \mathrm{e}^{\mathrm{K}} \Im\left[\mathrm{W} \overline{\mathrm{F}}_{\mathrm{I}}-\mathrm{G}^{\mathrm{l} \overline{\mathrm{j}}} \nabla_{\mathrm{l}} \mathrm{W} \bar{\nabla}_{\mathrm{j}} \overline{\mathrm{F}}_{\mathrm{I}}\right]
$$

To go further we use the identities,

$$
\begin{aligned}
\nabla_{i} \bar{W} & =0 \\
\nabla_{i} \bar{\nabla}_{\bar{j}} W & =G_{i \bar{j}} W
\end{aligned}
$$




$$
D_{i} \nabla_{j} W=\mathbf{i} C_{i j k} G^{k \bar{l}} \bar{\nabla}_{\bar{i}} \nabla_{l} W
$$

where the real symmetric coefficients $C_{i j k}$ are given as,

$$
C_{i j k}=\mathrm{e}^{\mathrm{K}}\left(\nabla_{\mathrm{i}} \mathrm{X}^{\mathrm{L}} \nabla_{\mathrm{j}} \mathrm{X}^{\mathrm{M}} \nabla_{\mathrm{k}} \mathrm{X}^{\mathrm{N}} \mathrm{C}_{\mathrm{LMN}}\right) ; \mathrm{C}_{\mathrm{LMN}} \equiv \partial_{\mathrm{L}} \partial_{\mathrm{N}} \partial_{\mathrm{M}} \mathrm{F}(\mathrm{X})
$$

and the operation $D_{i}$ involves the Christoffel connection as well as the Kähler connection, i.e. schematically, $D_{i} \equiv \nabla_{i}+\Gamma_{i}$. Now computing $D_{i} V=\nabla_{i} V$, via (12), the equation (14) takes the form,

$$
2 \nabla_{i} W \bar{W}+\mathbf{i} C_{i j k} G^{j \bar{m}} G^{k \bar{n}} \bar{\nabla}_{\bar{m}} \bar{W} \bar{\nabla}_{\bar{l}} \bar{W}=0
$$

Finally [8] reads $\nabla_{i} W$ from (20), substitutes it into the equations (17), (18) and solves the resulted equations to get the attractor values of the moduli, the result of course matches with that of [3].

To cook up our cake, we recast the equations (17), (18) and (20) in a new form which is closer to the original OSV language. The object of interest is a function defined on the complex structure moduli space of $X$ as,

$$
C \equiv 2 \mathbf{i} \mathrm{e}^{\mathrm{K}} \overline{\mathrm{W}}
$$

which enjoys the properties,

$$
\nabla_{i} C=0 ; \quad \bar{\nabla}_{\bar{i}} C=\bar{\partial}_{\bar{i}} C
$$

It is worth to note that the combinations $C X^{I}$ and $C F_{I}$ are invariant under the Kähler gauge transformations, $K(t, \bar{t}) \rightarrow K(t, \bar{t})-f(t)-\bar{f}(\bar{t})$.

In terms of (21), the equations (17), (18) and (20) take the following final forms,

$$
\begin{gathered}
2 C \partial_{i} \bar{C}-\mathbf{i} C_{i j k} G^{j \bar{n}} G^{k \bar{l}} \bar{\partial}_{\bar{n}} C \bar{\partial}_{\bar{l}} C=0 . \\
p^{i}=\Re\left[C X^{i}-G^{j \bar{l}} \bar{\nabla}_{\bar{l}} \bar{C} \nabla_{j} X^{i}\right] \\
q_{i}=\Re\left[C F_{i}-G^{j \bar{l}} \bar{\nabla}_{\bar{l}} \bar{C} \nabla_{j} F_{i}\right]
\end{gathered}
$$

with

$$
\begin{aligned}
& \Re\left[C X^{0}-G^{j \bar{l}} \bar{\nabla}_{\bar{l}} \bar{C} \nabla_{j} X^{0}\right]=p^{0} \\
& \Re\left[C F_{0}-G^{j \bar{l}} \bar{\nabla}_{\bar{l}} \bar{C} \nabla_{j} F_{0}\right]=q_{0} .
\end{aligned}
$$

We call (22) the "C-equations" and (23|26) the "charge equations" 5 . They, as a whole, form the most general attractor equations in the $\mathcal{N}=2$ effective theory of

\footnotetext{
${ }^{5}$ For applications in section 3, 25/26) are written separated from the other charge equations.
} 
II B $(A)$ on $C Y_{3}$. Having these equations at hand, one can safely forget the original definition of $C$, because they not only determine the attractor value of $C$ but also imply (21) as consequent relation. In fact to solve the equations (23), (24) and (22), the best way is treating them as a coupled system of $(4 n+2)$ nonlinear algebraic equations governing $(4 n+2)$ unknown variables. These variables are $\left(t^{i}, C ; \bar{\partial}_{\bar{i}} C\right)$ together with their complex conjugates. Although one is only interested in determining the attractor values of $t^{i}$, since the equations (22, 26) are strongly coupled, one needs to determine all of these variables.

The trivial solution of (22) is given simply as,

$$
\partial_{i} \bar{C}=\bar{\partial}_{\bar{i}} C=0
$$

which result at the well known BPS attractor equations [9],

$$
\begin{gathered}
p^{I}=\Re\left[C X^{I}\right] \\
q_{I}=\Re\left[C F_{I}\right] .
\end{gathered}
$$

As a warm up for the goal of this paper, we end this section with re-deriving the results of [3], [8] using the above set of equations. We set $p^{0}=0$ which is needed for the non-supersymmetric attractors and also avoids various conceptual and technical difficulties in the BPS case. Further without loss of generality, we can safely restrict the case to the simple STU model defined as,

$$
F=\frac{X^{1} X^{2} X^{3}}{X^{0}} ;(S, T, U) \equiv\left(t^{1}, t^{2}, t^{3}\right)
$$

and set $q_{i}=0$. As soon as one gets the final results, the dictionary to translate them to the general case of (11) with $q_{i} \neq 0$ is clear and simple, as we shall finally do. Working in the $X^{0}=1$ gauge and denoting the imaginary parts of the moduli with $t_{2}^{i}$, from (30), (111) and (19), one finds

$$
K=-\ln \left(-8 \mathrm{~S}_{2} \mathrm{~T}_{2} \mathrm{U}_{2}\right) \quad ; \quad \mathrm{C}_{\mathrm{ijk}}=\frac{-1}{8 \mathrm{~S}_{2} \mathrm{~T}_{2} \mathrm{U}_{2}}\left|\epsilon_{\mathrm{ijk}}\right| .
$$

It is easy to observe that the charge equations match with the conditions $p^{0}=q_{a}=0$ for,

$$
\begin{gathered}
t^{j}=\mathbf{i} t_{2}^{j} ; \forall j \\
C=\mathbf{i} C_{2}\left(S_{2}, T_{2}, U_{2}\right)
\end{gathered}
$$

Then denoting,

$$
C_{2, j}=\frac{\partial C_{2}}{\partial t_{2}^{j}}
$$


the nontrivial charge and C-equations take the form,

$$
\begin{gathered}
C_{2} C_{2,1}-\frac{T_{2} U_{2}}{S_{2}} C_{2,2} C_{2,3}=0 \\
C_{2} C_{2,2}-\frac{U_{2} S_{2}}{T_{2}} C_{2,3} C_{2,1}=0 \\
C_{2} C_{2,3}-\frac{S_{2} T_{2}}{U_{2}} C_{2,1} C_{2,2}=0 \\
\frac{p^{1}}{S_{2}}=S_{2} C_{2,1}-T_{2} C_{2,2}-U_{2} C_{2,3}-C_{2} \\
\frac{p^{2}}{T_{2}}=-S_{2} C_{2,1}+T_{2} C_{2,2}-U_{2} C_{2,3}-C_{2} \\
\frac{p^{3}}{U_{2}}=-S_{2} C_{2,1}-T_{2} C_{2,2}+U_{2} C_{2,3}-C_{2} \\
\frac{q_{0}}{S_{2} T_{2} U_{2}}=S_{2} C_{2,1}+T_{2} C_{2,2}+U_{2} C_{2,3}-C_{2}
\end{gathered}
$$

It turns out that if one varies the charges, the moduli space of solutions to the above set of equations is topologically disconnected. It is the union of two disjoint sectors, ' susy sector' and 'non-susy sector', which are characterized by the sign of $q_{0} \mathcal{D}$, with $\mathcal{D} \equiv p^{1} p^{2} p^{3}$.

Given the charge multiplet $\left(p^{1}, p^{2}, p^{3}, q_{0}\right)$, the susy solution of (31]37) reads as,

$$
\left(C_{2, j}=0 \quad ; \quad t_{2}^{j}=-\frac{p^{j}}{C_{2}} \quad ; \quad C_{2}^{2}=\frac{\mathcal{D}}{q_{0}}\right)
$$

while the non-susy solution is given by,

$$
\left(C_{2, j}=\frac{C_{2}}{t_{2}^{j}} \quad ; \quad t_{2}^{j}=-\frac{p^{j}}{2 C_{2}} \quad ; \quad C_{2}^{2}=-\frac{\mathcal{D}}{4 q_{0}}\right)
$$

To get the above attractor solutions, the easiest way is treating (31]37) as a coupled system of 7 algebraic equations governing 7 unknown variables $\left(t_{2}^{j}, C_{2}, C_{2, j}\right)$. A different but equivalent way is as follows. One first solves the equations (31]33) as a coupled system of 3 differential equations. Apart from the susy solution $C_{2}=$ const., this gives us a unique STU-symmetric non-susy solution as $C_{2}=r S_{2} T_{2} U_{2}$, with $r$ a constant value. Now substituting the non-susy solution of $C_{2}$ in the equations 
(34) 36 ) one gets, $\frac{S_{2}}{p 1}=\frac{T_{2}}{p 2}=\frac{U_{2}}{p 3}=A$, with, $A^{4}=\frac{-1}{2 \mathcal{D} r}$. Finally substituting all the above results in the equation (37), one obtains, $A= \pm \sqrt{\frac{-q 0}{\mathcal{D}}}$ and accordingly, $C_{2}= \pm \frac{1}{2} \sqrt{\frac{-\mathcal{D}}{q 0}}$. Totally, (39) is recovered.

We note that the attractor values of the susy and non-susy moduli are mapped to each other simply via an analytic continuation, $q_{0} \rightarrow-q_{0}$.

Now we lift the results (38) and (39) up to the general case of (10) with nonvanishing $q_{i}$, simply by mapping,

$q^{0} \rightarrow \hat{q}_{0}=q_{0}+\frac{1}{12} D^{i j}(p) q_{i} q_{j} ; t^{i} \rightarrow \hat{t}^{i}=t^{i}-\frac{1}{6} D^{i j}(p) q_{j} ; \mathcal{D} \rightarrow D(p)$.

It is worth to highlight the observation that the value of the entropy of the extremal black hole in the supergravity limit, given by the Bekenstein-Hawking formula, has the same value for both the BPS and non-BPS sectors [3]. It enjoys a simple relation with the value of the effective potential at the attractor point,

$$
S_{B H}=\pi V_{e f f}\left(t_{2}^{j *}\right)
$$

, which according to (38), (39) and (12) equals,

$$
S_{B H}=2 \pi \sqrt{|Q|}
$$

with $Q \equiv \hat{q}_{0} D(p)$ being the invariant charge. Finally one more comment about the non-susy attractor values is in order. Unlike the susy case where the condition (13) are necessary and sufficient for $V_{\text {eff }}$ to be minimum, for the non-susy solution of (14) the minimality condition should be checked independently. By expanding $V_{\text {eff }}$ around the non-susy attractor point one can check that 39$]$ is indeed a minimum [3].

\section{The mixed partition function}

\subsection{Recap of the OSV proposal}

Lets recap the steps that lead OSV to the mixed ensemble of [9]. They consider the BPS attractor equations (28) and (29) and first solve half of them, the magneticcharge attractors,

$$
C X^{I}=p^{I}+\mathbf{i} \frac{\phi^{i}}{\pi}
$$

Then using the fact that prepotential is homogeneous of order two, from (42) they define,

$$
\tilde{F}\left(p^{I}, \phi^{I}\right) \equiv C^{2} F\left(X^{I}\right)
$$


Further, ignoring holomorphic anomaly of the prepotential at higher genus levels ${ }^{6}$, they recast the entropy of BPS black holes as evaluated in [21,22], based on the Wald entropy formula [23], in the form of a Legendre transformation from the $O S V$ free energy,

$$
\mathcal{F}\left(p^{I}, \phi^{I}\right) \equiv-\pi \Im\left[\tilde{F}\left(p^{I}, \phi^{I}\right)\right]
$$

over half of its variables as follows,

$$
S(p, q)=\mathcal{F}-\phi^{I} \frac{\partial \mathcal{F}}{\partial \phi^{I}}
$$

with,

$$
q_{I}=-\frac{\partial \mathcal{F}}{\partial \phi^{I}}
$$

We note that (46) is equivalent with (29) and so completes the attractor equations. The result (45,46) suggests to interprate $\mathcal{F}$ as free energy of the BPS black hole carrying charges $\left(p^{I}, q_{I}\right)$. This corresponds to a mixed ensemble defined as,

$$
Z_{B H}=\mathrm{e}^{\mathcal{F}} \equiv \sum_{\mathrm{q}^{\mathrm{I}}} \Omega(\mathrm{p}, \mathrm{q}) \mathrm{e}^{-\mathrm{q}_{\mathrm{I}} \phi^{\mathrm{I}}}
$$

with $\Omega(p, q)$ being the black hole (index) degeneracy of states.

Finally according to the well known relation,

$$
F\left(C X^{I}, C^{2} \mathcal{W}^{2}\right)_{\left.\right|_{C^{2} \mathcal{W}^{2}=256}}=\frac{2}{\mathbf{i} \pi} F_{\text {top }}\left(t^{i}, g_{\text {top }}\right)_{\left.\right|_{g_{t o p}= \pm \frac{4 \pi \mathbf{i}}{C X^{0}}}}
$$

with $\mathcal{W}^{2}$ as the squared graviphoton field strength, OSV propose a beautiful connection between 4-dimensional BPS black holes and topological strings,

$$
\mathcal{F}=F_{\text {top }}+\bar{F}_{\text {top }} \quad \rightarrow \quad Z_{B H}\left(p^{I}, \phi^{I}\right)=\left|Z_{\text {top }}\left(t^{i}, g_{\text {top }}\right)\right|^{2}
$$

where

$$
t^{j}=\frac{p^{j}+\mathbf{i} \phi^{j} / \pi}{p^{0}+\mathbf{i} \phi^{0} / \pi} \quad ; \quad g_{t o p}= \pm \frac{4 \pi \mathbf{i}}{p^{0}+\mathbf{i} \phi^{0} / \pi}
$$

For concrete tests of the OSV proposal see [24-27], for some applications see [28,29] and for further developments of the original proposal see [30,31].

\footnotetext{
${ }^{6}$ For attempts to incorporate the holomorphic anomaly see [20] and the interesting proposal of [13].
} 


\subsection{OSV relation in the reduced ensemble}

An equivalent mixed ensemble can be obtained for BPS black holes if, instead of solving half of the attractor equations and keeping the second half untouched, one solves all but one of them and saves the last one, say $q_{0}$-equaton, for the Legendre transformation which leads to the black hole free energy. This results at a minimized version of the mixed ensemble, also supports for the relation (49) but with some other relations between the charge-potential variables and the topological variables, as compared to (50).

As a concrete example for the above claim, lets consider the BPS STU-example of section 2 and, for obtaining the black hole entropy as a Legendre transformation from some free energy, keep (37) unused. This way, from (31]36) one obtains,

$$
\left(C_{2, j}=0 \quad ; \quad t_{2}^{j}=-\frac{p^{j}}{C_{2}}\right)
$$

with the attractor value of $C_{2}$ undetermined at this stage. Moreover we define,

$$
\hat{\mathcal{F}}\left(\mathcal{D}, C_{2}\right) \equiv-\pi \Im\left[C^{2} F(X)\right]_{\mid[51]}=-\pi \Im[F(C X)]_{\mid[51]}
$$

which according to the relation (48) yields,

$$
\hat{\mathcal{F}}\left(\mathcal{D}, C_{2}\right)=2 \Re\left[F_{\text {top }}\left(t^{i}, g_{\text {top }}\right)\right]_{\left.\right|_{51]}}=2 \Re\left[F_{\text {top }}\left(t^{j}, g_{\text {top }}\right)\right]_{\left.\right|_{\left(g_{\text {top }}= \pm \frac{4 \pi}{C_{2}}, t^{j}=\frac{p^{j}}{\mathrm{i} C_{2}}\right)}} \cdot(
$$

From (152), (151) and (30) one gets,

$$
\hat{\mathcal{F}}\left(\mathcal{D}, C_{2}\right)=\pi \frac{\mathcal{D}}{C_{2}}
$$

Now lets perform a Legendre transformation on $\hat{\mathcal{F}}$ over the variable $C_{2}$ and call it $\mathcal{L}_{C_{2}}[\hat{\mathcal{F}}]$

$$
\mathcal{L}_{C_{2}}[\hat{\mathcal{F}}] \equiv \hat{\mathcal{F}}\left(\mathcal{D}, C_{2}\right)-C_{2} \frac{\partial \hat{\mathcal{F}}\left(\mathcal{D}, C_{2}\right)}{\partial C_{2}}
$$

Using the result (54) one gets,

$$
\mathcal{L}_{C_{2}}[\hat{\mathcal{F}}]=2 \pi \frac{\mathcal{D}}{C_{2}}
$$

It is turn to use (37). Substituting the results of (151) into (37) gives us the attractor value of $C_{2}$ which according to (38) is given by,

$$
C_{2}^{*}= \pm \sqrt{\frac{\mathcal{D}}{q_{0}}}
$$


Now comparing the value of entropy as given by (41) with (56) and (57) one deduces,

$$
S_{B H}=\mathcal{L}_{C_{2}^{*}}[\hat{\mathcal{F}}] \equiv \mathcal{L}_{C_{2}}[\hat{\mathcal{F}}]_{\left.\right|_{2} ^{*}}
$$

This suggests the identification of our BPS black hole free energy with $\hat{\mathcal{F}}\left(\mathcal{D}, C_{2}\right)$. Correspondingly the reduced mixed ensemble of this BPS black hole is proposed as,

$$
Z_{B H}(\mathcal{D}, C)=\mathrm{e}^{\hat{\mathcal{F}}\left(\mathcal{D}, \mathrm{C}_{2}\right)} \equiv \sum_{\mathrm{N}} \hat{\Omega}(\mathrm{ND}) \mathrm{e}^{-\mathrm{N} \mathrm{C}_{2}}
$$

with $N$ standing for the charge corresponding to $C_{2}$,

$$
N \equiv-\left.\frac{\partial \hat{\mathcal{F}}}{\partial C_{2}}\right|_{C_{2}^{*}}=\pi q_{0}
$$

and $\hat{\Omega}$ being the same as OSV degeneracy of states,

$$
\hat{\Omega}\left(\pi q_{0} \mathcal{D}\right)=\Omega(p, q) .
$$

Now according to (59) and (153) one recovers the OSV relation in the form,

$$
Z_{B H}(\mathcal{D}, C)=\left|Z_{\text {top }}\left(t^{i}, g_{\text {top }}\right)\right|_{\left(g_{\text {top }}= \pm \frac{4 \pi \mathbf{i}}{C}, t^{j}=\frac{p^{j}}{C}\right)}^{2}
$$

Finally in order of relaxing the gauge fixing condition $X^{0}=1$, we define

$$
\phi^{0} \equiv \pi \Im\left(C X^{0}\right)
$$

and then using the map (40) the generalization of the relation (62) is given by,

$Z_{B H}\left(D(p), \phi^{0}\right)=\mathrm{e}^{\frac{\pi^{2} \mathrm{D}(\mathrm{p})}{\phi^{0}}} \equiv \sum_{\pi \hat{\mathrm{q}}_{0}} \hat{\Omega}\left(\pi \mathrm{D}(\mathrm{p}) \hat{\mathrm{q}}_{0}\right) \mathrm{e}^{-\hat{\mathrm{q}}_{0} \phi^{0}}=\left|\mathrm{Z}_{\mathrm{top}}\left(\mathrm{t}^{\mathrm{j}}, \mathrm{g}_{\mathrm{top}}\right)\right|_{\left(\mathrm{g}_{\mathrm{top}}= \pm \frac{4 \pi^{2}}{\phi^{0}}, \mathrm{t} \mathrm{j}=\frac{\pi \mathrm{p}^{\mathrm{j}}}{\mathrm{i} \phi^{0}}\right)}($

which is the case for all the BPS black holes with vanishing D6-brane charge in the supergravity limit.

Here to highlight the equivalence of the ensemble (64) with the OSV ensemble (47), it is worth to explicitly calculate the OSV free energy (44) for the BPS black hole carrying charges $\left(p^{i}, q_{i}, q_{0}\right)$ in the supergravity defined by (11). It is evaluated as,

$$
\mathcal{F}\left(p^{I}, \phi_{I}\right)=\frac{\pi^{2} D}{\phi^{0}}-\frac{3 D_{a b} \phi^{a} \phi^{b}}{\phi^{0}}
$$

which, since $C_{2}=\frac{\phi^{0}}{\pi}$ in the gauge $X^{0}=1$, differs from (154) by the second term at the right hand side of (65). However (54) and (65), although presenting different functions, define equivalent free energies for the black hole under consideration, as seen by the equalities,

$$
\mathcal{L}_{\left\{\phi^{I}\right\}}[\mathcal{F}]=\mathcal{L}_{C_{2}}[\hat{\mathcal{F}}] \quad ; \quad \phi_{\left.\right|_{q_{I}}=-\frac{\partial \mathcal{F}}{\partial \phi^{I}}}^{0}=\phi_{\hat{q}_{0}=-\frac{\partial \hat{\mathcal{F}}}{\partial \phi^{0}}}^{0}
$$

Although compared to the OSV relations (49) and (50), the relation (64) is more restricted since it does not incorporate neither the higher-gins terms nor the $D 6$ brane charge, the procedure which leaded to the ensemble (64) is of importance for us. We shall follow the same approach for the case of non-supersymmetric black holes in the next subsection. 


\subsection{Partition function of the non-supersymmetric attractors}

To find the partition function of the non-susy attractors in the supergravity limit, we begin with the non-susy sector of the STU model with $\left(p^{0}, q_{i}\right)=0$, as discussed in section 2, and after deriving the partition function, map the result to the general case of (11) with nonvanishing $q_{i}$, via (40).

The procedure is similar to what was done in the previous subsection for the susy sector. If we keep the equation (37) unsolved, the equations (31,36) together, for the non-susy sector, imply,

$$
\left(C_{2, j}=\frac{C_{2}}{t_{2}^{j}} \quad ; \quad t_{2}^{j}=-\frac{p^{j}}{2 C_{2}}\right)
$$

Therefore defining,

$$
\hat{\mathcal{F}}_{n s}\left(\mathcal{D}, C_{2}\right) \equiv-\pi \Im\left[C^{2} F(X)\right]_{[66}=-\pi \Im[F(C X)]_{[66}
$$

From (67), (66) and (30) one gets,

$$
\hat{\mathcal{F}}_{n s}\left(\mathcal{D}, C_{2}\right)=\pi \frac{\mathcal{D}}{8 C_{2}}
$$

We perform a Legendre transformation on $\hat{\mathcal{F}}_{n s}$ over the variable $C_{2}$ and call it $\mathcal{L}_{C_{2}}\left[\hat{\mathcal{F}}_{n s}\right]$,

$$
\mathcal{L}_{C_{2}}\left[\hat{\mathcal{F}}_{n s}\right] \equiv \hat{\mathcal{F}_{n s}}\left(\mathcal{D}, C_{2}\right)-C_{2} \frac{\partial \hat{\mathcal{F}_{n s}}\left(\mathcal{D}, C_{2}\right)}{\partial C_{2}}
$$

which with (68) equals,

$$
\mathcal{L}_{C_{2}}\left[\hat{\mathcal{F}}_{n s}\right]=\pi \frac{\mathcal{D}}{4 C_{2}}
$$

Further, lets compute the attractor value of $\mathcal{L}_{C_{2}}\left[\hat{\mathcal{F}}_{n s}\right]$ and compare with $S_{B H}$ as given by (41). Substituting the results of (66) into (37), the attractor value of $C_{2}$ is found to be,

$$
C_{2}^{*}= \pm \frac{1}{2} \sqrt{-\frac{\mathcal{D}}{q_{0}}}
$$

so that,

$$
\mathcal{L}_{C_{2}^{*}}\left[\hat{\mathcal{F}}_{n s}\right]= \pm \frac{\pi}{2} \sqrt{-q_{0} \mathcal{D}}
$$

Now comparing (72) with (41) one observes,

$$
S_{B H}=4 \mathcal{L}_{C_{2}^{*}}\left[\hat{\mathcal{F}}_{n s}\right]
$$


Thus defining,

$$
\mathcal{F}_{n s . B H}\left(\mathcal{D}, C_{2}\right) \equiv 4 \hat{\mathcal{F}_{n s}}\left(\mathcal{D}, C_{2}\right)
$$

one obtains,

$$
S_{n s . B H}=\mathcal{L}_{C_{2}^{*}}\left[\mathcal{F}_{n s}\right] .
$$

which completes the procedure. Thus the mixed partition function of this non-susy black hole is proposed as,

$$
Z_{n s . B H}(\mathcal{D}, C)=\mathrm{e}^{\mathcal{F}_{\text {ns. BH }}\left(\mathcal{D}, \mathrm{C}_{2}\right)} \equiv \sum_{\mathrm{M}} \mathrm{d}(\mathrm{M} \mathcal{D}) \mathrm{e}^{-\mathrm{M} \mathrm{C}_{2}}
$$

with $M$ regarded as the charge corresponding to $C_{2}$,

$$
M \equiv-\left.\frac{\partial \mathcal{F}_{n s . B H}}{\partial C_{2}}\right|_{C_{2}^{*}}=-2 \pi q_{0}
$$

and $\mathrm{d}(\mathrm{MD})$ as the black hole degeneracy of states carrying charges $\left(p^{1}, p^{2}, p^{3}, q_{0}\right)$. Finally defining $\phi^{0} \equiv \pi \Im\left(C X^{0}\right)$ to relax the gauge fixing condition $X^{0}=1$, and using the map (40), we generalize the above partition function to the case of general non-supersymmetric black holefor non-supersymmetric black holes attractors in the supergravity limit as follows,

$$
Z_{n s . B H}\left(D(p), \phi^{0}\right)=\mathrm{e}^{\frac{\pi^{2} \mathrm{D}(\mathrm{p})}{2 \phi^{0}}} \equiv \sum_{-2 \pi \hat{\mathrm{q}}_{0}} \mathrm{~d}\left(-2 \pi \mathrm{D}(\mathrm{p}) \hat{\mathrm{q}}_{0}\right) \mathrm{e}^{2 \hat{\mathrm{q}} 0 \phi^{0}}
$$

\section{Open questions}

Here we briefly mention two problems which call for further investigations:

1. The result for $d(p, q)$ as derived from the partition function (2) can be checked with an independent counting of the black hole degeneracy of states in a given set up. However for non-supersymmetric black holes it seems pretty nontrivial to find a set up in which such a counting is possible.

2. The proposed partition function (22) is restricted to the supergravity limit. It is interesting to look for the partition function of non-supersymmetric black holes beyond this limit. Again compared to BPS black holes it is a more nontrivial job to do. For example the role played by the D-terms and the holomorphic anomaly is mainly unknown for the case of non-supersymmetric black holes.

\section{Acknowledgement}

This is our pleasure to thank A. Dabholkar and S. P. Trivedi for useful discussions. A. T. would like to thank the Department of Theoretical Physics at TIFR for warm hospitality. We are especially grateful to S. Minwalla and C. Vafa for their insightful comments about the draft. 


\section{References}

[1] A. Sen, "Black hole entropy function and the attractor mechanism in higher JHEP 0509, 038 (2005) arXiv:hep-th/0506177.

[2] K. Goldstein, N. Iizuka, R. P. Jena and S. P. Trivedi, "Non-supersymmetric attractors," arXiv:hep-th/0507096.

[3] P. K. Tripathy and S. P. Trivedi, "Non-supersymmetric attractors in string theory," arXiv:hep-th/0511117.

[4] R. Kallosh, "New attractors," JHEP 0512, 022 (2005) arXiv:hep-th/0510024.

[5] G. W. Gibbons, R. Kallosh and B. Kol, "Moduli, scalar charges, and the first law of black hole thermodynamics," Phys. Rev. Lett. 77, 4992 (1996) arXiv:hep-th/9607108.

[6] S. Ferrara, G. W. Gibbons and R. Kallosh, "Black holes and critical points in moduli space," Nucl. Phys. B 500, 75 (1997) arXiv:hep-th/9702103.

[7] B. Chandrasekhar, S. Parvizi, A. Tavanfar and H. Yavartanoo, "Nonsupersymmetric attractors in $\mathrm{R}^{* *} 2$ gravities," arXiv:hep-th/0602022

[8] R. Kallosh, N. Sivanandam and M. Soroush, "The non-BPS black hole attractor equation," arXiv:hep-th/0602005.

[9] H. Ooguri, A. Strominger and C. Vafa, "Black hole attractors and the topological string," Phys. Rev. D 70, 106007 (2004) arXiv:hep-th/0405146.

[10] A. Dabholkar, F. Denef, G. W. Moore and B. Pioline, "Exact and asymptotic degeneracies of small black holes," JHEP 0508, 021 (2005) arXiv:hep-th/0502157.

[11] A. Dabholkar, F. Denef, G. W. Moore and B. Pioline, "Precision counting of small black holes," JHEP 0510, 096 (2005) arXiv:hep-th/0507014.

[12] D. Shih and X. Yin, "Exact black hole degeneracies and the topological string," arXiv:hep-th/0508174.

[13] G. Lopes Cardoso, B. de Wit, J. Kappeli and T. Mohaupt, "Black hole partition functions and duality," arXiv:hep-th/0601108.

[14] S. Parvizi and A. Tavanfar, "Minimal redefinition of the OSV ensemble," arXiv:hep-th/0508231.

[15] S. Gukov, C. Vafa and E. Witten, "CFT's from Calabi-Yau fourfolds," Nucl. Phys. B 584, 69 (2000) [Erratum-ibid. B 608, 477 (2001)] arXiv:hep-th/9906070. 
[16] S. Ferrara, R. Kallosh and A. Strominger, "N=2 extremal black holes," Phys. Rev. D 52, 5412 (1995) arXiv:hep-th/9508072.

[17] A. Strominger, "Macroscopic Entropy of $N=2$ Extremal Black Holes," Phys. Lett. B 383, 39 (1996) arXiv:hep-th/9602111.

[18] S. Ferrara and R. Kallosh, "Supersymmetry and Attractors," Phys. Rev. D 54, 1514 (1996) arXiv:hep-th/9602136.

[19] S. Ferrara and R. Kallosh, "Universality of Supersymmetric Attractors," Phys. Rev. D 54, 1525 (1996) arXiv:hep-th/9603090.

[20] E. P. Verlinde, "Attractors and the holomorphic anomaly," arXiv:hep-th/0412139.

[21] G. Lopes Cardoso, B. de Wit and T. Mohaupt, "Macroscopic entropy formulae and non-holomorphic corrections for Nucl. Phys. B 567, 87 (2000) arXiv:hep-th/9906094.

[22] G. Lopes Cardoso, B. de Wit and T. Mohaupt, "Area law corrections from state counting and supergravity," Class. Quant. Grav. 17, 1007 (2000) arXiv:hep-th/9910179.

[23] R. M. Wald, "Black hole entropy in the Noether charge," Phys. Rev. D 48, 3427 (1993) arXiv:gr-qc/9307038.

[24] C. Vafa, "Two dimensional Yang-Mills, black holes and topological strings," arXiv:hep-th/0406058.

[25] A. Dabholkar, "Exact counting of black hole microstates," Phys. Rev. Lett. 94, 241301 (2005) arXiv:hep-th/0409148.

[26] M. Aganagic, H. Ooguri, N. Saulina and C. Vafa, "Black holes, q-deformed 2d Yang-Mills, and non-perturbative topological Nucl. Phys. B 715, 304 (2005) arXiv:hep-th/0411280.

[27] M. Aganagic, D. Jafferis and N. Saulina, "Branes, black holes and topological strings on toric Calabi-Yau manifolds," arXiv:hep-th/0512245.

[28] S. Gukov, K. Saraikin and C. Vafa, "A stringy wave function for an $\mathrm{S}^{* *} 3$ cosmology," arXiv:hep-th/0505204.

[29] S. Gukov, K. Saraikin and C. Vafa, "The entropic principle and asymptotic freedom," arXiv:hep-th/0509109.

[30] M. Aganagic, A. Neitzke and C. Vafa, "BPS microstates and the open topological string wave function," arXiv:hep-th/0504054. 
[31] R. Dijkgraaf, R. Gopakumar, H. Ooguri and C. Vafa, "Baby universes in string theory," arXiv:hep-th/0504221. 